\title{
ON THE CONCEPT OF CONNECTEDNESS
}

\author{
Nikita Shekutkovski
}

\begin{abstract}
Definition of quasicomponents by coverings is given and It is shown the equivalence with standard definition. Intrinsic definition of pointed 1-movability is given, that uses only coverings. It is shown that two definitions coincide.
\end{abstract}

\section{DEFINITION OF CONNECTEDNESS BY COVERINGS}

The standard definition of connectedness from the books of topology is:

Definition 1.1. Suppose $A$ and $B$ are nonempty subsets of the topological space $X . A$ and $B$ are separated if $\bar{A} \cap B=\varnothing=A \cap \bar{B} . X$ is conncted, if $X$ cannot be expressed as union of two separated sets.

This definition of connectedness is given in the beginning of $20^{\text {th }}$ century by Riesz and Hausdorff.

The main minus of the definition is that definition is given by negative sequence.. Here will be presented another definition based on coverings.

Suppose $\mathrm{F}$ is a family of subsets of $X$, and $x$ and $y$ are two points in $X$. A chain in $\mathrm{F}$ from $x$ and $y$ is a finite sequence $F_{1}, F_{2}, \ldots, F_{n}$ of members of $\mathrm{F}$ such that $x \in F_{1}, y \in F_{n}$ and $F_{i} \cap F_{i+1} \neq \varnothing$, for $1 \leq i \leq n-1$.

Definition 1.2. Suppose $X$ is a topological space. $X$ is connected if for any two points $x$ and $y$ in $X$ and any open covering of $X$ there is a chain of members of the covering from $x$ to $y$

2010 Mathematics Subject Classification. Primary: 54D05

Key words and phrases. Connectedness, coverings, quasicomponents, path connectedness, pointed 1-movability, proximate path connectedness 
Proposition 1.1. The two definitions of connectedness coincide.

Proof. Suppose $X$ is a topological space, $X$ is connected by Definition 1, and $\mathrm{U}$ is an open covering of $X$. For two points $x$ and $y$ in $X$, define $A$ to be the set of all points $p$ of $X$ such that there is a chain in $\boldsymbol{U}$ from $x$ to $p$ and define $B$ to be the set of all points $p$ of $X$ such that there is a chain in $\boldsymbol{U}$ from $y$ to $p$.

If we suppose that $A \cap B=\varnothing$ then it must be $\bar{A} \cap B=\varnothing$ (on the contrary if $b \in \bar{A}$ and $b \in B$, then there will be a chain $\boldsymbol{u}$ from $x$ to $b$ i.e. $b \in A$ ). Similarly it must be $A \cap \bar{B}=\varnothing$ and we will obtain a contradiction.

It follows $A \cap B \neq \varnothing$, i.e. there exists a chain in $\boldsymbol{u}$ from $x$ to $y$.

Now, suppose $X$ is connected by Definition 2. If $X$ can be expressed as union of two separated sets $A$ and $B$, then both sets are open, and if we choose points $x \in A$ and $y \in B$, then in the open covering $\{A, B\}$ there is no chain from $x$ to $y$ It follows $X$ cannot be expressed as union of two separated sets i.e. $X$ is connected by definition 1 .

In the case of compact metric spaces Definition 2 can be simplified i.e.

Definition 1.2'. Suppose $X$ is a compact metric spaces. $X$ is connected if for any two points $x$ and $y$ in $X$, and any $r>0$ there is a finite chain of open $r-$ balls from $x$ to $y$

Proof. Def 2) $\Rightarrow$ Def 2') Take the covering of $X$ consisting of all oen $r$-balls. By Definition 2 we can choose a finite chain of open $r$-balls from $x$ to $y$.

For compact metric spaces we will prove the converse i.e. Def 2') $\Rightarrow$ Def 2) . Take an open covering $\boldsymbol{U}$ of $X$. By Lebesgue number Lemma we can choose $r$ $>0$ such that all balls with radius $r$ and centers in points of $X$ are contained in some meber of $\boldsymbol{U}$. By Definition 2' there is a finite chain $r$-balls from $x$ to $y$, say $B_{r}\left(x_{1}\right), B_{r}\left(x_{2}\right), \ldots, B_{r}\left(x_{n}\right)$. Since, there exist members $U_{1}, U_{2}, \ldots, U_{n}$ of the covering $\boldsymbol{U}_{\text {such that }}$

$$
B_{r}\left(x_{1}\right) \subseteq U_{1}, \quad B_{r}\left(x_{2}\right) \subseteq U_{2} \quad, \ldots, \quad B_{r}\left(x_{n}\right) \subseteq U_{n}
$$

The finite sequence $U_{1}, U_{2}, \ldots, U_{n}$ is a chain in $\boldsymbol{u}$ from $x$ to $y$.

The Definition 2' is in fact the same with definition of connectedness given by Cantor in the period from 1879 till 1884 : 
(Cantor definition of connectedness) Space is connected if for any two points $x$ and $y$ and any $r>0$ there is a finite number of points

$$
x=x_{0}, x_{1}, x_{2}, \ldots, x_{n+1}=y
$$

such that

$$
d\left(x_{i}, x_{i+1}\right)<\varepsilon .
$$

Of course, if compact metric space is connected by Definition 2' i.e. for two points $x$ and $y$ there is a chain $B_{r}\left(x_{1}\right), B_{r}\left(x_{2}\right), \ldots, B_{r}\left(x_{n}\right)$ of $r$-balls from $x$ to $y$, then for the points $x=x_{0}, x_{1}, x_{2}, \ldots, x_{n+1}=y$ is satisfied $d\left(x_{i}, x_{i+1}\right)<\varepsilon$.

On the other hand if compact metric space is connected by Cantor definition, and for two points $x$ and $y$ there is a finite number of points $x=x_{0}, x_{1}, x_{2}, \ldots$, $x_{n+1}=y$ such that $d\left(x_{i}, x_{i+1}\right)<\varepsilon$, then $B_{r}\left(x_{1}\right), B_{r}\left(x_{2}\right), \ldots, B_{r}\left(x_{n}\right)$ is a chain of $r$-balls from $x$ to $y$,

The definition of connectedness by coverings has advantages in some situations, for example a simpler definition of qasicomponents.

A quasicomponent of a point $x$ is ussualy defined as the intersection of all clopen (= open and closed) sets containing the point $x$.

Theorem 1.1. Suppose $X$ is a topological space, $x$ and $y$ points in $X$. The following statements are equivalent

1) $x$ and $y$ belong to the same quasicomponent

2) for any covering of $X$ there is a chain of members of the covering from $x$ to $y$

Proof: 1$) \Rightarrow 2$ ) Suppose $\mathrm{x}$ and y belong to the same quasicomponent and there is a covering $\boldsymbol{U}$ such that there is no chain in $\boldsymbol{U}$ from $x$ to $y$. Define $A$ to be the set of all points $p$ of $X$ such that there is a chain in $\boldsymbol{U}$ from $x$ to $p$. Then $A$ is open. Also, $A$ is closed since for any point $p \in \bar{A}$, there is a chain in $\boldsymbol{U}$ from $x$ to $p$. If we put $B=X \backslash A$ then $A$ and $B$ are clopen, $x \in A$ and $y \in B, \mathrm{a}$ contradiction with the fact that they belong to the same quasicomponent.

$2) \Rightarrow 1)$ Suppose for any covering of $X$ there is a chain of members of the covering from $x$ to $y$. If we suppose that there exists a clopen set $A, x \in A$ and $y \notin A$. Then in the open covering $\{A, X \backslash A\}$ there is no chain from $x$ to $y$. 
By the previous Theorem, the quasicomponent of a point $x$ can be defined by

Definition 1.3. The quasicomponent of a point $x$ consists of all points $p$ such that for all open coverings $\boldsymbol{U}$ there is a chain in $\boldsymbol{U}$ from $x$ to $p$.

Since the notions of component and quasicomponent coincide for compact metric spaces the above definition is definition of a component in compact metric spaces.

\section{A CONNECTIVITY NOTION BETWEEN CONNECTEDNESS AND PATH CONNECTEDNESS}

The notion of path connectedness is very natural i.e. a topological space $X$ is connected if for any two points $x$ and $y$ there is a path from $x$ to $y$. Historically it appeared before the notion of connectedness

The subject of this section is a kind of strong connectivity that stays between connectedness and path connectedness.

At the end of sixties of $20^{\text {th }}$ century was introduced the notion of pointed 1movability by K. Borsuk. The original definition uses embeddings of compact metric spaces in Hilbert cube.

Instead of Borsuk definition of pointed 1- movability here is presented modified definition given in [3] and [4] under name joinability.

In [3] is proved that the two notions coincide.

Definition 2.1. Suppose $X \subseteq Q$ is a continuum and $x, y \in X$. We say that $h:[0,1] \times[0, \infty) \rightarrow Q$ is approximative $X$-path between $x$ and $y$ if

1) $h(0, s)=x$ and $h(1, s)=y$ for each $s$ in $[0, \infty)$

2) for each neighborhood $U$ of $X$ in $Q$ there is $s \geq 0$ such that $h([0,1] \times[s, \infty)) \subseteq U$

$X$ is joinable (pointed 1-movable) if between any two points in $\mathrm{X}$ there is an approximative $X$-path.

Here will be presented another definition of this notion that uses only coverings of the space. The notion was first presented in [5] and used to study connectivity properties of chain recurrent set in a dynamical system. 
For collections $\boldsymbol{u}$ and $\boldsymbol{v}$ of subsets of $X, \boldsymbol{u} \prec \boldsymbol{v}$ means that $\boldsymbol{u}$ refines $\boldsymbol{v}$, i.e. each $U \in \boldsymbol{U}$ is contained in some $V \in \mathcal{v}$. If $U \in \boldsymbol{U}$, then the star of $U$ is the set $\operatorname{St}(U, \boldsymbol{u})=\{W \in \boldsymbol{U} \mid W \cap U \neq \varnothing\}$ and by St $\boldsymbol{U}$ is denoted the collection of all $\operatorname{St}(U, \boldsymbol{u}), U \in \boldsymbol{U}$.

By a covering we understand a covering consisting of open sets.

Definition 2.2. Suppose $\mathcal{V}$ is a covering of $Y$. A function $f: X \rightarrow Y$ is $\mathcal{V}$ continuous at point $x \in X$, if there exists a neighborhood $U_{x}$ of $x$, and $V \in \mathcal{V}$, such that $f\left(U_{x}\right) \subseteq V$.

A function $f: X \rightarrow Y$ is $\mathcal{V}$-continuous, if it is $\mathcal{V}$-continuous at every point $x \in X$.

Definition 2.3. The functions $f, g: X \rightarrow Y$ are $V$-homotopic, if there exists a function $F: X \times I \rightarrow Y$ such that:

1) $F: X \times I \rightarrow Y$ is $s t(\mathcal{V})$ - continuous

2) $F: X \times I \rightarrow Y$ is $\mathcal{V}$ - continuous at all points of $X \times \partial I$

3) $F(x, 0)=f(x), F(x, 1)=g(x)$

Here $I=[0,1]$ is the unit interval. If $f: X \rightarrow Y$ is $\mathcal{V}$ - continuous, then $f: X \rightarrow Y$ is $\boldsymbol{W}$ - continuous for any $\boldsymbol{W}$ such that $\boldsymbol{v} \prec \boldsymbol{W}$. By this, since $V \prec s t(\mathcal{V})$, if the function $F: X \times I \rightarrow Y$ in above definition is is $V$-continuous then conditions 1) and 2) are satisfied,

A $V$ - continuous path is $V$ - continuous function $k:[a, b] \rightarrow X$

Further on, we will consider only compact metric spaces. In this case it is enough to consider only finite coverings.

A sequence of finite coverings, $v_{11} \succ v_{2} \succ \ldots$ of a compact metric space such that for any covering $\mathcal{V}$, there exists $n$, such that $v_{\succ} v_{n}$ we call a cofinal sequence of finite coverings.

A proximate path is defined by a cofinal sequence of finite coverings $v_{1} \succ$ $v_{2} \succ \ldots$ and a sequence $\left(k_{n}\right)$, of $v_{n}$ - continuous paths $k_{n}: I \rightarrow X$, and for all indices $m \geq n, k_{n}$ and $k_{m}$ are $V_{n}$ - homotopic relative $\{0,1\}$. 
Definition 2.4. $X$ is proximate path connected if for any two points $\mathrm{x}$ and $\mathrm{y}$ there is a proximate path $\left(k_{n}\right)$ from $x$ to $y$, i.e. $k_{n}(0)=x, k_{n}(1)=y$ for all integers $n$.

By the definition above it is clear that path connectedness implies proximate path connectedness, and proximate path connectedness imples connectedness.

To the end of the section the following theorem will be proven.

Theorem 2.1. Pointed 1-movable in the sense of Borsuk $\Leftrightarrow$ strongly connected.

First we will prove direction $(\Rightarrow)$. We need the following:

A covering $\boldsymbol{V}$ of $M$ in $X$ is called regular if it satisfies the following conditions:

1) If $V \in V$, than $V \cap M \neq \varnothing$.

2) If $U, V \in V$ and $U \cap V \neq \varnothing$, than $U \cap V \in V$.

About the condition 1) see definition of proper covering, ([2], Definition 8.1., p. 249), while the condition 2) together with 1) shows that $v$ is a regular family relative to $M$ in the sense of [2] (Definition 3.5. p. 262).

For a covering $v$ of $M$ we introduce the notation $|\boldsymbol{v}|=\underset{V \in \mathcal{V}}{\cup V}$. For a regular finite covering $V$ of $M$, we define a function $r_{v}:|\boldsymbol{V}| \rightarrow M$ in the following way:

For points $y \in M$ we put $r_{\boldsymbol{v}}(y)=y$.

For points $y \in|V| M$, by induction we can choose the smallest member $V \in$ $v$ such that $y \in V$, then choose a fixed point $[V] \in V \cap M$ and put $r_{v}(y)=[V]$.

The defined function $r_{v}$ is $v$-continuous.

Now, suppose $v_{1} \succ v_{2} \succ \ldots$ is a cofinal sequence of regular finite coverings of $X$ in the Hilbert cube $Q$.

For any two points $x, y \in X$, there is an approximative $X$-path $h:[0,1] \times[0, \infty) \rightarrow Q$ between $x$ and $y$.

Then, there is $s_{j} \geq 0$ such that $h\left([0,1] \times\left[s_{j}, \infty\right)\right) \subseteq\left|\boldsymbol{v}_{j}\right|$. Define $h_{j}: I \rightarrow Q$ by

$$
h_{j}(t)=h\left(t, s_{j}\right)
$$


and define a $v_{j}=$ path $k_{j}: I \rightarrow X$ by

$$
k_{j}(t)=r \mathcal{V}_{j} h_{j}(t)
$$

To prove that $\left(k_{j}\right)$, is a proximate path we have to prove that for all indices $j, k_{j}$ and $k_{j+1}$ are $v_{j}$ - homotopic relative $\{0,1\}$.

Define $H_{j}: I \times\left[s_{j}, s_{j+1}\right] \rightarrow Q$ by

$$
H_{j}(t, s)=h(t, s)
$$

and define a $\mathcal{V}_{j}$ - continuous function $K_{j}: I \times\left[s_{j}, s_{j+1}\right] \rightarrow X$ by

$$
K_{j}(t, s)=r_{v_{j}} H_{j}(t, s)
$$

Then

$$
K_{j}\left(t, s_{j}\right)=h_{j}\left(t, s_{j}\right)=k_{j}(t)
$$

and

$$
K_{j}\left(t, s_{j+1}\right)=h_{j}\left(t, s_{j+1}\right)=k_{j+1}(t)
$$

Also

$$
K_{j}(0, s)=r_{v_{j}} H_{j}(0, s)=r_{v_{j}} h(0, s)=r_{v_{j}}(x)=\mathrm{x}
$$

and similarly

$$
K_{j}(1, s)=y \text {. }
$$

These proves that $\left(k_{j}\right)$ is a proximate path from $x$ to $y$.

To prove the the direction $(\Leftarrow)$ we need some preliminaries:

Definition 2.3. If $\boldsymbol{U}$ is an covering of $X$, we say that points $x . y \in X$ are $\boldsymbol{u}-$ near if there is $U \in \mathcal{u}$, such that $x . y \in U$.

Let $V$ be a covering of $Y$. Two functions $f, g: X \rightarrow Y$ are $V$ - near, if for any $x \in X$ the points $f(x)$ and $g(x)$ are $\mathcal{V}$-near.

For a metric space $X$ with a metric $d$ a notion of " $r$ - near" can be defined.

Definition 2.3'. $X$ a metric space with metric $d$. For a given $r>0$, we say that points $x . y \in X$ are $r$ - near if $d(x . y)<r$

Two functions $f, g: Y \rightarrow X$ are $r$-near, if for any $y \in Y$ the points $f(y)$ and $g(y)$ are $r-$ near i.e. $d(f(y) . g(y))<r$. 
(Lemma of Ho, [1] ) Let $X$ be a paracompact space, and $C$ a convex subset of a normed linear space $L$. For any $r$ - continuous function $f: X \rightarrow C$ there exists a $r$-near function $g: X \rightarrow C$ which is continuous.

The proof of this Lemma [1] can be easily modified with additional assumption that in a point $x_{0} \in X$, to be satisfied $f\left(x_{0}\right)=g\left(x_{0}\right)$.

For the proof of direction $(\Leftarrow)$, suppose $\left(k_{j}\right)$ is a proximate path from $x$ to $y$ over a cofinal sequence $v_{1} \succ v_{2} \succ \ldots$ of finite coverings of $X$ in Hilbert cube $Q$.

The proximate path $\left(k_{j}\right)$ from $x$ to $y$, consists of $v_{j}$ - continuous paths $k_{j}: I \rightarrow X$, and for all indices $k_{j}$ and $k_{j+1}$ are $v_{j}$ - homotopic relative $\{0,1\}$, i.e there exists $v_{j}$-continuous function $K_{j}: I \times[0,1] \rightarrow X$ such that

$$
K_{j}(t, 0)=k_{j}(t)
$$

and

$$
K_{j}(t, 1)=k_{j+1}(t)
$$

Also

$$
K_{j}(0, s)=\mathrm{x}
$$

and

$$
K_{j}(1, s)=y
$$

By induction on $j$ we will construct continuous functions $H_{j}: I \times[0,1] \rightarrow Q$. such that $H_{j}(0, s)=x, H_{j}(1, s)=y$, and such that $H_{j-1}(1, s)=H_{j}(0, s)$.

Suppose continuous functions $H_{1}, H_{2}, \ldots, H_{j-1}$ are constructed. By Lemma of Ho, for $K_{j}: I \times[0,1] \rightarrow X$ there is a $v_{j}$ - near continuous function $H_{j}: I \times[0,1] \rightarrow Q$ such that $H_{j}(0, s)=\mathrm{x}, H_{j}(1, s)=y$ and $H_{j}(t, 0)=H_{j-1}(t, 1)$.

By the last condition we can glue these continuous function and obtain a continuous function $h:[0,1] \times[1, \infty) \rightarrow Q$ by formula

$$
h(t, s)=H_{j}(t, j+s)
$$

for $s \in I$ and $s \in[j, j+1]$. This continuous function satisfies

1) $h(0, s)=x$ and $h(1, s)=y$ for each $s$ in $[1, \infty)$ 
2) for each neighborhood $U$ of $X$ in $Q$ there is $s \geq 0$ such that $h([0,1] \times[s, \infty)) \subseteq U$

i.e. $h:[0,1] \times[1, \infty) \rightarrow Q$ is an approximative $X$-path between $x$ and $y$

Example. Dyadic solenoid i of Van Dantzig/Vietoris s not pointed I-movable/ joinable/proximatepath connected.

Dyadic solenoid is the inters of members of the sequence $T_{1} \supset T_{2} \supset \ldots \supset T_{\mathrm{n}}$ $\supset \ldots$ The first member of sequence $T_{1}$ is a solid torus. Each next member of this sequence, $T_{i}$, is a solid torus twice twisted and embedded in the previous $T_{i-1}$

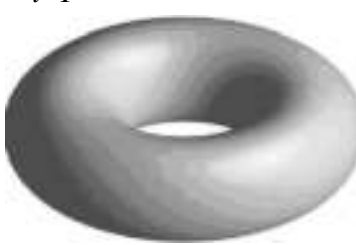

$T_{1}$

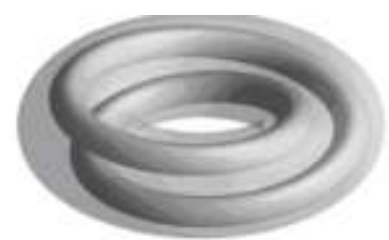

$T_{2}$ twice twisted and embedded in $T_{1}$

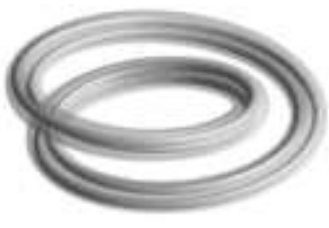

$T_{2}$

Fig. 1

References

[1] C. Ho, On a stability theorem for the fixed point theorem, Fund. Math. 64 (1969), 181-188

[2] S. Eilenberg, N. Steenrod, Foundations of Algebraic Topology, vol. 15, Princeton University Press, New Jersey, 1952

[3] J. Krasinkiewicz and P. Minc, Generalized paths and pointed 1-movability, Fund. Math. 104 (1979), 141-153

[4] Piotr Minc, Remainders of arcwise connected compactifications of the plane, Top. Appl. Volume 154, Issue 8, 15 April 2007, Pages 1592-1599

[5] N. Shekutkovski, One property of components of chain recurrent set, Regular and Chaotic Dynamics, Vol. 20 , Issue 2, (2015), 184-188

[6] N. Shekutkovski, Intrinsic shape - The proximate approach, Filomat 29:10 (2015) $2199-2205$.

Instutute of Mathematics,

Faculty of Natural sciences and Mathematics,

University of St. Cyril and Methodius,

Skopje, Republic of Macedonia

E-mail address: nikita@pmf.ukim.mk 